\title{
Crying newborns could breathe into droplets with virus:the discussion of the possibility of intrapartum transmission
}

\author{
Rui-Hong Xue ${ }^{1}$ and Hefeng Huang ${ }^{2}$ \\ ${ }^{1}$ International Peace Maternity and Child Health Hospital, School of Medicine, Shanghai \\ Jiao Tong University, Shanghai, China \\ ${ }^{2}$ International Peace Maternity and Child Health Hospital, School of Medicine,
}

October 20, 2020

\section{Crying newborns could breathe into droplets with virus:the discussion of the possibility of intrapartum transmission}

Rui-Hong Xue ${ }^{1}$, He-Feng Huang ${ }^{2,3,4,5}$

${ }^{1}$ Department of Obstetrics and Gynecology, International Peace Maternity and Child

Health Hospital, School of Medicine, Shanghai Jiao Tong University, Shanghai, China

${ }^{2}$ Center of Reproductive Medicine, International Peace Maternity and Child Health Hospital, School of Medicine, Shanghai Jiao Tong University, Shanghai, China

${ }^{3}$ Institute of Embryo-Fetal Original Adult Disease Affiliated to Shanghai Jiao Tong University School of Medicine, Shanghai Jiao Tong University, Shanghai, China

${ }^{4}$ Shanghai Key Laboratory of Embryo Original Diseases, Shanghai, China

${ }^{5}$ Shanghai Municipal Key Clinical Specialty, Shanghai, China

Correspondence: He-feng Huang, E-mail:huanghefg@hotmail.com;Postal address: No.910 Hengshan Road, Shanghai, China. Postal code: 200030. Work phone: +86-021-64070434

The author report no conflict of interest.

Word Count: 463

Funding: None

Sir,

We read with great interests the article by Anat Schwartz and colleagues, entitled "Detection of SARS-CoV-2 in vaginal swabs of women with acute SARS-CoV-2 infection: a prospective study". In their findings, of the 35 patients sampled, $2(5.7 \%)$ had a positive vaginal RT-PCR for SARS-CoV-2, one was pre-menopausal and the other was a post-menopausal woman, they did not detect the presence of viral colonization in the vagina in five pregnant women. Although the possibility of false positive results could not be ruled out, the prospective study contained important information, with inclusion of the participant women in both reproductive and non-reproductive years.

The detections of virus in the vagina and breast milk in pregnant women have been reported in several studies, and concluded that vaginal delivery or breast milk feeding might be low risk, for the major sampled vagina and breast milk had negative RT-PCR for SARS-CoV-2. 
However, the transmission of virus by droplets between the mother and newborn might have been seriously underestimated. SARS-CoV-2 spreads through contact (via larger droplets and aerosols), and longer-range transmission via aerosols, especially in conditions where ventilation is poor. Its high infectivity, combined with the susceptibility of unexposed populations to a new virus, creates conditions for rapid community spread. A retrospective study of 42 pregnant women with COVID-19, two women had a new diagnosis of COVID-19 infection in the postpartum period and breast-fed without a surgical mask; both newborns had a positive test for COVID-19 infection at day 1 and 3, respectively. In another case after vaginal delivery, the newborn of an infected woman had a positive test and the first test for SARS-CoV-2 was equivocal a few hours after delivery, and positive 3 days later, whereas, in 10 cases infected women with elective caesarean section for conditions related to COVID-19 respiratory syndrome, no newborn was diagnosed with positivity to SARS-Cov-2. Although the authors concluded that vaginal delivery was associated with a low risk of intrapartum SARS-Cov-2 transmission to the newborn, we would like to emphasize the virus concentrations in the delivery room between vaginal birth and Caesarean delivery might be very different.

COVID-19 infected mother may release lots of droplets containing virus in the room when using deep breathing and abdominal pressure in the process of vaginal delivery, even if taking a mask, COVID-19 characters as strong infectivity, rapid and wide spread. The neonate might breathe the virus into the lung with crying, and the first crying might be the important time-point for the neonates exposing to COVID-19. And they are the main differences in COVID-19 infection risks between vaginal and cesarean deliveries, we should not solely consider whether the vagina secretion and breast milk contain virus. The importance of possible intrapartum transmission and prevention in COVID-19 infected pregnant women should be considered in clinic.

\section{Contributions}

HF-H was responsible for the design and supervision of the work and have made critical revision. RH-X wrote the manuscript.

\section{Sources of funding}

None

\section{Disclosure of interests}

The author of this work declare no potential conflicts of interest relevant to this article.

\section{Ethics approval}

The manuscript was approved by the ethics committee on human research. Review Board: (GKLW) 2020-05 On March 15, 2020. 